\title{
Uso de plantas medicinais: Histórico e relevância
}

\author{
Use of medicinal plants: History and relevance \\ Uso de las plantas medicinales: Historia y relevancia
}

\section{Resumo}

O uso de plantas pode ser considerado uma das formas mais antigas de práticas terapêuticas. Com base nessa afirmação, o presente artigo buscou descrever os principais fatos históricos relativos à utilização de plantas medicinais desde a pré-história até a atualidade. O objetivo do trabalho foi a realização de um levantamento bibliográfico sobre o histórico de uso de plantas medicinais e a sua relevância frente a implementação de políticas públicas de saúde. A pesquisa foi realizada de setembro a outubro de 2020, sendo analisados artigos e dissertações presentes em bases de dados específicas (Schoolar Google, Science Direct e Pubmed). Ao término do estudo pode-se perceber a fundamental importância dos saberes populares presentes em diferentes culturas, como fator essencial a construção e implementação políticas sólidas de saúde, de âmbito nacional, como o Sistema Único de Saúde (SUS) e a Política Nacional de Plantas Medicinais e Fitoterápicos (PNPMF).

Palavras-chave: Etnobotânica; Etnofarmacologia; Terapia alternativa; SUS. 


\begin{abstract}
The use of plants can be considered one of the oldest forms of therapeutic practices. Based on this statement, this article sought to describe the main historical facts regarding the use of medicinal plants from prehistory to the present. The objective of the work was to carry out a bibliographic survey on the history of use of medicinal plants and their relevance to the implementation of public health policies. The research was carried out from September to October 2020, analyzing articles and dissertations present in specific databases (Schoolar Google, Science Direct and Pubmed). At the end of the study, it is possible to perceive the fundamental importance of popular knowledge present in different cultures, as an essential factor in the construction and implementation of solid national health policies, such as the Unified Health System (SUS) and the National Plant Policy Medicinal and Herbal Medicine (PNPMF).
\end{abstract}

Keywords: Ethnobotany; Ethnopharmacology; Alternative therapy; SUS.

\title{
Resumen
}

El uso de plantas puede considerarse una de las formas más antiguas de prácticas terapéuticas. Partiendo de esta afirmación, este artículo pretendió describir los principales hechos históricos relacionados con el uso de las plantas medicinales desde la prehistoria hasta la actualidad. El objetivo del trabajo fue realizar un estudio bibliográfico sobre la historia del uso de las plantas medicinales y su relevancia para la implementación de políticas de salud pública. La investigación se llevó a cabo de septiembre a octubre de 2020, analizando artículos y disertaciones presentes en bases de datos específicas (Schoolar Google, Science Direct y Pubmed). Al final del estudio, es posible percibir la importancia fundamental del conocimiento popular presente en diferentes culturas, como factor esencial en la construcción e implementación de políticas nacionales de salud sólidas, como el Sistema Único de Salud (SUS) y la Política Nacional de Plantas Medicinales y Herbales (PNPMF).

Palabras clave: Etnobotánica; Etnofarmacología; Terapia alternativa; SUS.

\section{Introdução}

Com a maior biodiversidade do mundo, sendo responsável por aproximadamente $20 \%$ da flora mundial, o Brasil exibe notoriedade no que diz respeito ao desenvolvimento de novas metodologias terapêuticas baseadas em produtos naturais (Calixto, 2003). Entre esses produtos, estão às plantas medicinais, que segundo a Organização Mundial de Saúde correspondem a todas as espécies vegetais que apresentam em uma ou mais partes, substâncias químicas capazes de desempenhar atividades farmacológicas, auxiliando na cura e/ou tratamento de várias doenças (OMS, 1998).

A atividade farmacológica em plantas deve-se a presença de princípios ativos capazes de desempenhar inúmeras atividades biológicas, exercendo um papel fundamental frente ao processo de saúde-doença. (Filho \& Yunes, 1998; Phillipson, 2001).

No contexto da utilização de plantas medicinais, a abordagem etnodirigida, ou seja, aquela em que a seleção das espécies se baseia no conhecimento popular, têm contribuído com a síntese de novos produtos farmacêuticos, uma vez que este atual como facilitador no processo de bioprospecção em plantas (Albuquerque \& Hanazaki, 2006; Brandão et al, 2010). Estima-se que apenas nos últimos 25 anos, $77,8 \%$ de todos os medicamentos anticancerígenos descobertos são provenientes de produtos naturais (Nogueira; Cerqueira \& Soares, 2010).

Com registros datando desde 60.000 anos a.C, estando presentes em diversas culturas, como a Egípcia, Hindu, Persa, Grega e os povos da américa pré-colombiana as plantas medicinais podem ser consideradas uma das formas mais antigas de práticas terapêuticas (Rocha et al, 2015, Saraiva et al, 2015), sendo o somatório de todas essas contribuições culturais às responsáveis pelos avanços científicos atuais na área de produtos naturais (Viegas; Bolzani \& Barreiro, 2006).

A associação dos conhecimentos indígenas, europeus e africanos no Brasil, foi responsável pela transformação da fitoterapia em uma prática sociocultural, integrando-a a cultura popular brasileira (Ibiapina et al, 2014). A facilidade na absorção dos conhecimentos fitoterápicos pelos povos brasileiros deve-se principalmente ao alto custo e alta toxicidade dos medicamentos sintéticos e inacessibilidade de acesso aos serviços de saúde pelas comunidades, o que promove uma maior busca pelos medicamentos de origem vegetal (Batista \& Valença, 2012).

Entre os grandes avanços relativos ao uso das plantas medicinais no Brasil, estão à inserção da Fitoterapia no Sistema 
Único de Saúde (Brasil, 1988) e a criação da Política Nacional de Plantas Medicinais e Fitoterápicos (Figueiredo; Dantas Gurgel \& Gurgel Junior, 2014), assim como a criação de políticas públicas, como as Práticas Integrativas e Complementares (Ferreira et al, 2020), que denotam à construção de uma política nacional capaz de abranger às populações mais carentes, assim como garantem a eficácia frente a validação científica do uso de plantas medicinais (Bruning; Mosegui \& Vianna, 2012).

O presente estudo teve como objetivo a análise e construção de um levantamento bibliográfico acerca dos usos de plantas medicinais ao longo do tempo, e como tais conhecimentos contribuíram para a construção de políticas públicas de saúde atuais.

\section{Metodologia}

Esta revisão foi realizada por meio de uma busca realizada de setembro a outubro de 2020, realizada por meio de bancos de dados de busca especializados (Schoolar Google, PubMed, e Science Direct) usando várias combinações das seguintes palavras-chave, em inglês e português: uso, medicinal, plantas, história, brasil, mundo, bioprospecção, biodiversidade, etnofarmacologia, sistema único de saúde, rename, saúde, ciência e cultura. A seleção dos manuscritos baseouse nos critérios de inclusão: 1) levantamentos históricos e etnobotânicos sobre uso de plantas medicinais em diferentes culturas. 2) impacto da utilização de plantas no sistema de saúde. 3) biodiversidade como provedor de recursos biológicos. Foram excluídos artigos que apresentassem divergência frente aos critérios selecionados. Nesta pesquisa, foram identificados 178 artigos, destes foram selecionados 60 artigos, pois os demais não atenderam aos critérios de inclusão ou foram indexados em duas ou mais bases de dados e foram considerados apenas uma vez.

A escrita do artigo foi dividida em três diferentes momentos, sendo o primeiro momento constituído como uma revisão sistemática da biodiversidade. O segundo momento constituído pelo histórico do uso de plantas medicinais, disposto em ordem cronológica. E o terceiro sendo composto pela importância da utilização de plantas medicinais ao longo da história e sua importância frente a construção e implementação de políticas públicas de saúde.

\section{Resultados e Discussão}

\subsection{Biodiversidade: Conceitos Básicos}

De acordo com o decreto $\mathrm{n}^{\mathrm{o}}$ 2.519, a Convenção da Diversidade Biológica (CDB), define biodiversidade como a variabilidade de seres vivos presentes nos ecossistemas terrestres ou aquáticos, englobando a diversidade presente dentro de espécies, entre espécies e entre ecossistemas (Brasil, 1992). Os estudos sobre a biodiversidade não só são importantes para a compreensão de seus processos evolutivos e ecológicos, como também detém relevância quanto ao uso de seus recursos naturais. Segundo Lévêque (1999) "sob o termo 'recursos biológicos' identificamos os componentes da biodiversidade que têm uma utilização direta, indireta ou potencial para a humanidade".

O termo "biodiversidade" geralmente é associado apenas a diferença entre seres vivos, ou seja, diferentes espécies. No entanto, cabe ressaltar que a biodiversidade não deve ser interpretada como um conjunto de elementos individuais, e sim pela soma de todas as diferenças entre as espécies, suas interações em diferentes níveis, assim como suas variações químicas e genéticas. Dessa forma, a biodiversidade se divide em três diferentes componentes: diversidade ecológica, diversidade genética e a diversidade de espécies (Amorim \& Chow, 2016).

Em termos conceituais, a diversidade ecológica compreende as relações entre os organismos com o ambiente, e entre seus semelhantes, possibilitando uma heterogeneidade de comunidades e relações ecológicas (Amorim \& Chow, 2016). Nesse contexto, a diversidade ecológica abrange às comunidades e os ecossistemas. Cabe salientar, que a interação planta-ambiente é um dos fatores responsáveis pela diversidade de constituintes químicos presentes nas plantas (Rodrigues \& Guedes, 2006). 
A diversidade de espécies corresponde ao número de espécies diferentes, ou seja, a riqueza de espécies (Amorim \& Chow, 2016). Estima-se que no mundo existam mais de 279 mil espécies de plantas superiores, no entanto grande parte dessa diversidade ainda não foi analisada do ponto de vista terapêutico (Peixoto \& Morim, 2003; Pereira Júnior, 2005; Silva, A. F, 2014).

A diversidade genética nada mais é do que a variabilidade em termos de material genético entre diferentes espécies e diferentes indivíduos de uma mesma espécie. É a diversidade genética junto aos fatores ambientais, como luz, disponibilidade de nutrientes e temperatura, as responsáveis pelas diferenças morfológicas, fisiológicas e químicas presentes nos organismos (Amorim \& Chow, 2016).

\subsection{Plantas Medicinais: Uso Popular E Farmacológico}

Dentre os componentes que constituem a biodiversidade, estão as plantas medicinais. Para a Organização Mundial de Saúde (OMS), planta medicinal é "todo e qualquer vegetal que possui, em um ou mais órgãos, substâncias que podem ser utilizadas com fins terapêuticos ou que sejam precursores de fármacos semissintéticos" (OMS, 1998). As ações terapêuticas realizadas pelas plantas medicinais devem-se aos princípios ativos presentes nas diferentes partes da planta (sementes, raízes, flores, frutos e folhas), sendo eles os responsáveis pela resposta fisiológica em organismos vivos (Phillipson, 2001).

Atualmente os princípios ativos de origem vegetal têm sido foco de vários estudos químicos e farmacológicos que visam elucidar dúvidas em relação a utilização de plantas medicinais como medida terapêutica. Esses estudos afirmam que a partir observação, descrição e experimentação é possível relacionar espécies medicinais à suas respectivas atividades biológicas (Farnsworth, 1994). Entre às atividades biológicas encontradas em plantas medicinais, pode-se citar: atividade antifúngica, antimicrobiana, antiespasmódica, anti-inflamatória, antialérgica, antitumoral, analgésica, antioxidante, fotoprotetora, entre outros (Filho \& Yunes, 1998).

A etnofarmacologia, ou seja, o estudo das preparações medicinais utilizadas no processo saúde-doença pelas comunidades tradicionais, possui grande importância por fornecer informações essenciais à elaboração de estudos químicos e farmacológicos sobre plantas, objetivando o desenvolvimento de medicamentos ou isolamento de princípios ativos de interesse industrial (Miranda Et al, 2013). Em decorrência do alto custo e inúmeros efeitos colaterais presentes em medicamentos sintéticos, assim como a inacessibilidade da população aos serviços de saúde, têm sido crescente a insatisfação da população, que acaba por recorrer a métodos terapêuticos alternativos, como a fitoterapia (Batista \& Valença, 2012).

Entende-se por fitoterapia, o estudo das plantas medicinais e suas aplicações terapêuticas, desconsiderando o uso de princípios ativos isolados, mesmo que de origem vegetal (Ministério Da Saúde, 2006). A fitoterapia pode ser configurada como uma ferramenta integrativa útil, devido a suas características únicas, como a fácil acessibilidade, eficácia, baixo custo e a facilidade de incorporação em preparos caseiros, podendo assim ser utilizada para suprir a ausência de medicamentos sintéticos, ou até mesmo em substituição a estes (Santos et al, 2011).

Filho e Yunes (1998) afirmam que há uma maior probabilidade de se encontrar atividades biológicas em plantas quando a sua utilização na medicina popular é considerada, do que quando estas são escolhidas ao acaso, dado este corroborado por (Oliveira; Simoes \& Sassi, 2006), que afirmam que 85\% dos fitoterápicos têm origem nos arredores de comunidades tradicionais. As atividades biológicas presentes nas plantas são de grande importância para à manutenção da saúde em países em desenvolvimento, por este ser a única forma de acesso dessas populações à saúde básica (Oliveira Et al, 2006; Rodrigues \& Gomide, 2019).

O Brasil se destaca não apenas por sua flora megadiversa, mas por possuir um enorme potencial farmacológico, consequência direta da grande diversidade biológica presente no país - aproximadamente 56 mil espécies - equivalente a 19\% da flora mundial (Giulietti et al, 2005), distribuídas em seus seis macros biomas: Amazônia, Cerrado, Caatinga, Pampas, 
Pantanal e Mata Atlântica (IBGE, 2013).

O conhecimento sobre o uso de plantas medicinais no Brasil deve-se aos povos primitivos, especialmente aos indígenas, que detinham o conhecimento sobre as ações benéficas causadas pelo uso das plantas (Viegas; Bolzani \& Barreiros, 2006). Fato este, indispensável para o descobrimento de várias substâncias tóxicas ou terapêuticas ao longo do tempo. A partir dessas análises, é possível afirmar que os avanços científicos atuais na área de produtos naturais devem-se ao somatório das contribuições das mais diferentes culturas ao redor do mundo (Viegas; Bolzani \& Barreiros, 2006). Ainda no que concerne o uso de plantas medicinais no âmbito popular, este por si só não é capaz de validar cientificamente as plantas medicinais como potenciais produtos fitoterápicos, fazendo-se assim necessária a constatação da eficácia destes por meio de dados experimentais (Matos, 1994).

\subsection{Histórico De Uso Dos Produtos Naturais}

\section{Pré-história}

Desde a pré-história, o homem já fazia uso dos recursos naturais para realização de suas atividades básicas, como a alimentação. Entre esses recursos estão as plantas, que sempre foram utilizadas com essa finalidade, que ao longo do tempo passaram a ser utilizadas na confecção de roupas, em construções, como abrigo, ferramentas, entre outros (Braga, 2011). Acredita-se que o uso medicinal das plantas tenha tido início mediante observações de suas características únicas, como modificações estruturais durante às estações do ano, e seu poder de regeneração mediante injúrias (Rocha Et al, 2015).

A utilização das plantas na pré-história baseia-se em uma perspectiva mágico-simbólica, atribuindo aos espíritos e aos elementos naturais a responsabilidade pelas doenças. Nesse período era comum a utilização das plantas medicinais em rituais religiosos que buscavam aproximar o ser humano do divino, almejando à cura as inúmeras moléstias. Cabe ressaltar que na pré-história a religião era um ponto importante na determinação do cuidado (Barros, 2002; Cruz, 2014).

Supõe-se que a utilização das plantas com finalidade terapêutica durante a pré-história tenha sido transmitida oralmente, sendo difundida aos povos antigos (Rocha Et al, 2015). Escrituras arqueológicas confirmam a utilização de espécies psicoativas no Timor (Indonésia), com cerca de 11.000 anos a.C (Pelt, 2004). Na América do Sul existem registros do uso de coca (Erythroxylum coca Lam) datando 5.000 anos a.C (Pinto Et al, 2002). O uso das plantas medicinais no combate a doenças, se faz presente na América pré-colombiana através das culturas Maias, Astecas, Olmeca e Tolteca, que detinham o conhecimento sobre o uso medicinal de espécies como a Ipecacuanha (Cephaelis ipecacuanha (Brot.) Tussac), e a quina (Chincona sp.) (Pinto et al, 2002).

\section{Antiguidade}

A antiguidade marca um momento de transição da medicina mágico-simbólica para a empírico-racional, que compreende a busca por explicações não sobrenaturais para o surgimento do universo e da vida, assim como para o processo saúde-doença. Hipócrates constatou a relação entre o homem/ambiente, formulando a teoria dos humores, no qual a explicação para o processo saúde-doença consistia numa relação entre água, terra, fogo e ar, sendo a doença resultado do desequilíbrio entre os elementos, e a saúde relativa ao equilíbrio entre estes (Cruz, 2014; Hoffmann \& Anjos, 2018).

Depositado no "British Museum", e escritos em placa de barro com caracteres cuneiformes, estão alguns dos primeiros documentos Sumérios e Babilônicos, feitos por ordem do rei assírio Ashurbanipal, datando aproximadamente 3000 anos a.C (Cunha, 2005). Dentre esses escritos, o mais conhecido é o código Hamurabi que descreve o uso de espécies vegetais bioativas, como o ópio, o gálbano e o meimendro no tratamento de doenças (Cunha, 2005).

Com o surgimento da escrita, a propagação dos conhecimentos eruditos torna-se comum. A partir da análise de trabalhos posteriores, infere-se que os manuscritos mais antigos sobre a utilização de plantas medicinais tenham cerca de 5000 
anos a.C (Pires, 1984). O conhecimento erudito mais antigo são os escritos de Shen Nong (2800 anos a.C.), que relata o uso de diversas plantas medicinais no tratamento de várias patologias (Almeida, 2011, 2019; Firmo et al, 2011). Não menos importante, o Papiro de Ebers (1500. a.C), que descreve o uso de 150 espécies medicinais, se consolida como um dos documentos mais importantes da cultura médica (Almeida, 2011, 2019; Firmo et al, 2011).

Outras escrituras que se destacam são a Susrata-samhiita (600 anos a.C), na Índia antiga, onde são catalogadas 700 espécies vegetais, e a Ayurveda, que mencionam plantas medicinais que são utilizadas até os tempos atuais, como o acônito (Aconitum napellus L.) e o aloé (Aloe sp.) (Monteiro \& Brandelli, 2017). Na China se faz presente a primeira farmacopeia datando 659 anos d.C, abrangendo a medicina tradicional chinesa, com registros de cerca de 2500 anos a.C (Almeida, 2011; Firmo et al, 2011).

Através da incorporação dos recursos terapêuticos Persas, os Gregos são responsáveis por grande parte do conhecimento fitoterápico. Foram eles os responsáveis pela produção de acervos bibliográficos contendo a descrição e utilização de diversos recursos terapêuticos (Ronan, 1987). Dentre os médicos mais relevantes da época se encontram o Hipócrates (460-370 a.C), considerado o pai da medicina moderna, fazia uso de 364 espécies vegetais bioativas (Firmo et al, 2011). Teophrastus (371-287 a.C), responsável pela elaboração do livro "História das plantas", no qual descreve precisamente as plantas quanto a sua morfologia, e seu uso tóxico e medicinal (Firmo et al, 2011) e Galeno (129-199 a.C), pioneiro de algumas das formas farmacêuticas como a infusão, as pastilhas, as pílulas, e os clisteres, até hoje utilizadas na medicina contemporânea (FIRMO et al, 2011). Por fim, o Dióscorides considerado pai da farmagnosia, que realizou o levantamento das plantas na Penísula Ibérica, África e Síria criando mais tarde o tratado "De matéria medica", um marco na história da fitoterapia e uma referência no mundo Romano e Árabe, que vigorou até o final da Idade Média (Firmo et al, 2011; Cabral \& Pita, 2015).

\section{Idade Média}

Os principais escritos presentes da Idade Média foram "De Re Medica" de John Mesue (850 DC), "Canon Medicinae" de Avicenna (980-1037) e "Liber Magnae Collectionis Simplicum Alimentorum Et Medicamentorum" de Ibn Baitar (1197 1248), juntas essas obras compreendem 1000 espécies vegetais catalogadas (Petrovska, 2012; Rocha et al, 2015).

A história afirma que a partir da inserção de novas plantas de caráter fitoterápico e o desenvolvimento de novas tecnologias a partir da incorporação da medicina grega e indiana, os árabes foram capazes de ao longo dos anos estabelecer sua medicina a níveis mais complexos, se consolidando como referência para as gerações posteriores (Almeida, 2011; Saad, 2014). Ainda no que concerne à complexidade da medicina árabe, essa se deu principalmente pela incorporação de medicamentos compostos, ou seja, foram introduzidas juntamente às plantas medicinais, os medicamentos de origem animal, mineral ou outros medicamentos vegetais (Petrovska, 2012).

Foi Paracelso (1493-1541) o responsável pela preparação de medicamentos a partir do extrato bruto de plantas e substâncias minerais (Gomes \& Reis, 2000). Esse entendimento junto a observação e sua crença na "Signature doutrinae", que consistia na suposição que Deus sinalizava substâncias bioativas nas plantas, com a finalidade de dar dicas sobre o seu uso em certas doenças. Por exemplo, a morfologia das folhas de erva de São-João (Hypericum perforatum L) remetem a picadas, determinando assim o seu uso no tratamento deste (Petrovska, 2012). Muito provavelmente foram esses estudos sobre respostas terapêuticas frente a substâncias extraídas de espécies vegetais, os responsáveis pelo primeiro entendimento sobre moléculas bioativas (Almeida, 2011).

\section{Idade Moderna}

Na idade média, o monopólio do conhecimento sobre plantas medicinais, seu cultivo e uso terapêutico foram 
concedidas aos monastérios, sendo estes os responsáveis pela retomada gradual da evolução terapêutica por meio da utilização dos conhecimentos greco-latinos junto ao uso das plantas por eles cultivadas (Cunha, 2005; Petrovska, 2014). Na idade moderna, por volta do século $\mathrm{XV}$, grande parte do conhecimento médico estava em posse das instituições religiosas, sendo uma das mais conceituadas a Ordem Benedinita, que partir da medicina monástica, ou seja, aquela realizada no interior dos monastérios, serviu de facilitador para abertura de várias enfermarias, boticas e jardins botânicos (Dias, 2005; Medeiros, 2009).

Todas as construções relativas a práticas terapêuticas desde a Idade Média até o início da Idade Moderna baseavam-se em observação e proximidade com a natureza, no entanto o Renascentismo foi capaz de recuperar influências da antiguidade greco-romana a partir da consolidação de valores, como o antropocentrismo, humanismo e individualismo, resultando em avanços de cunho econômico, político, social e técnico-científico (Costa, 2017). Com nomes como Francis Bacon (1561-1626) que através do método experimental e indutivo objetivava garantir ao homem o controle de sua realidade e René Descartes (1596-1650), com sua obra "Discurso do Método" detém relevante importância no surgimento do método científico racional (SOUZA, 2019).

\section{Idade contemporânea}

Segundo Walker (2013), o uso de plantas como uma potencialidade terapêutica antecede o período colonial, uma vez que o conhecimento é adquirido a partir da incorporação de práticas convencionais de diversas tribos indígenas. A riqueza de suas espécies vegetais e seus usos tem sido constantemente evidenciada desde a época do descobrimento. Pero Vaz de Caminha, em sua carta descreve a utilização de espécies vegetais, como o urucum (Bixa orelana L.) e seu potencial uso como corante (Filgueiras \& Peixoto, 2002). O uso da flora brasileira na alimentação, no tratamento de doenças e como cosméticos também foi observado por Pedro Álvares Cabral (Walker, 2013). Não menos importante, os jesuítas através da elaboração de registros e tratados médicos sobre a flora brasileira, tornaram-se pioneiros da farmacopeia brasileira (Calainho, 2005).

Apesar de constituir um aspecto fundamental no tratamento de doenças, a fitoterapia ao longo do século XX foi colocada em segundo plano, fato este que se deve ao desenvolvimento dos processos industriais mediante a síntese de novos medicamentos (Neto \& Caetano, 2005).

As plantas medicinais fizeram-se presente ao longo da história e ainda integram boa parte dos procedimentos clínicos tradicionais, mesmo com o avanço da medicina alopática na metade do século XX, contudo ainda existem desafios no uso deste pelas comunidades carentes que variam desde a dificuldade de acesso a estabelecimentos médico-hospitalares à obtenção de exames e medicação. $\mathrm{O}$ grande uso das plantas medicinais em substituição aos medicamentos sintéticos em países em desenvolvimento além das razões anteriores, também se deve aos costumes próprios de cada população, a facilidade de acesso as plantas e por fim a propaganda midiática em torno de produtos à base de espécies vegetais bioativas (Veiga; Pinto \& Maciel, 2005).

Cabe salientar que a redução do uso dos fitoterápicos em detrimento dos fármacos sintéticos ao longo da evolução industrial também é relativa ao controle dos processos envolvidos na produção dos medicamentos, que para os sintéticos são controlados, diferentemente dos naturais onde sua produtividade depende do clima, solo, pragas e doenças (Veiga et al, 2005). Apesar disso, no final do século XX houve uma retomada no uso das plantas medicinais em substituição aos medicamentos sintéticos, que se deve tanto as reações adversas causadas por estes, quanto ao desconhecimento dos possíveis efeitos negativos causados pelas plantas, visto que o conhecimento tradicional se baseia na crença de que "tudo que é natural não faz mal" (Sheldon, 1997; Batista \& Valença, 2012). Essa redescoberta pode ser evidenciada pelo aumento do número de estabelecimentos com ênfase na comercialização de produtos naturais, como também pelo reaparecimento de ocupações ditas tradicionais, como os raizeiros, em grandes mercados (Luz, 2005). 


\subsection{Plantas Medicinais no Sistema Único de Saúde (SUS)}

A década de 1960 e 1970, no Brasil, foram marcadas pelo surgimento de inúmeros movimentos sociais que buscavam melhorias das condições de saúde, entre eles o "Movimento Social Urbano" e a "Reforma Sanitária Brasileira", sendo este último um dos responsáveis por alicerçar a democratização do acesso à saúde, uma vez que suas ideias retratam a saúde como um direito social (Luz, 2005; Paim, 2008; Figueiredo, 2018).

Um dos grandes avanços relativos ao uso de plantas medicinais na idade contemporânea foi a politização e a democratização do acesso à saúde, proporcionada pelo Sistema Único de Saúde (SUS). Como citado no artigo 198 da Constituição Federal (CF), “a saúde é direito de todos e dever do Estado, garantido mediante políticas sociais e econômicas que visem à redução do risco de doença e de outros agravos e ao acesso universal e igualitário às ações e serviços para sua promoção, proteção e recuperação". Com o reconhecimento da saúde como um direito social, foi instituída a criação do SUS, constituída por uma rede regionalizada e hierarquizada, seguindo três diretrizes básicas: [1] descentralização, com direção única em cada esfera do governo, [2] o atendimento integral, priorizando as atividades preventivas, evitando prejuízos aos serviços assistenciais e [3] a participação da sociedade (Brasil, 1988).

Em consonância à constituição, em 19 de setembro de 1990 foi criada a Lei Orgânica da Saúde, que regula às ações e serviços de saúde e estabelece princípios do SUS, entre eles a universalidade, integralidade e igualdade de assistência à saúde sem preconceitos ou privilégios de qualquer natureza (Brasil, 1988). Devido ao princípio de descentralização de serviços, que retira das mãos da união a responsabilidade de ser o único responsável pela criação e implantação de políticas públicas relativas à saúde, os estados e munícipios obtiveram mais autonomia, fomentando a implantação de ações tais como as Práticas Integrativas e Complementares (PICS), que contempla a homeopatia, medicina tradicional chinesa/acupuntura e medicina antroposófica e a fitoterapia (Ferreira et al, 2020).

Por meio do decreto presidencial de $\mathrm{n}^{\circ} 5.813$ de 22 de junho de 2006 foi criada a Política Nacional de Plantas Medicinais e Fitoterápicos (PNPMF), objetivando a garantia da eficácia e segurança frente ao uso das plantas medicinais. Visando ainda a ampliação dos serviços oferecidos, a criação de um marco regulatório para produção e distribuição de plantas e fitoterápicos, o fortalecimento da agricultura familiar, o uso sustentável dos recursos naturais e por fim, o incentivo ao desenvolvimento científico e a indústria farmacêutica brasileira (Ministério Da Saúde, 2006).

A PNPMF junto as PICS, fomentaram debates acerca das oportunidades, importância, dificuldades, facilidades e vantagens da implementação da fitoterapia no SUS, assim como às perspectivas de como ocorreria o processo. Evidencia-se assim que as construções acerca de uma política sólida de âmbito nacional sobre o uso das plantas medicinais devem-se tanto aos atores sociais envolvidos no processo, como os agentes da saúde, pesquisadores e gestores, quanto a junção do conhecimento empírico-científico, que tem sido essencial no desenvolvimento de novas práticas terapêuticas (Figueiredo et al, 2014).

Em 2010, por meio da portaria $\mathrm{n}^{\circ} 886$ foi criada e implementada no SUS, a primeira assistência farmacêutica baseada na utilização de plantas medicinais, o Programa Farmácia Viva, que tinha como objetivo a produção de fitoterápicos acessíveis à população, considerando todas as etapas de sua fabricação, desde o cultivo, coleta, processamento, armazenamento de plantas medicinais, manipulação, dispensação de preparações magistrais até oficinas de plantas medicinais e produtos fitoterápicos (SANTOS et al, 2011).

Por meio do decreto 7.508/2011 foi regulamentada a Relação de Nacional de Medicamentos Essenciais (RENAME), onde em 2014 foram elencados 12 medicamentos fitoterápicos (Brasil, 2011, 2015). Apesar dos Estados e Municípios investirem em políticas públicas de atenção à saúde primária, ainda se faz necessário investimento em educação permanente dos profissionais de saúde, uma vez que às informações sobre os fitoterápicos existem, mas não são corretamente repassadas aos pacientes. 
É importante salientar que através da compreensão do contexto histórico do uso de produtos naturais, que são hoje possíveis a criação e implementação de políticas de âmbito nacional na área da saúde, como a PNPMF, que por si só é capaz de simbolizar um avanço frente a validação científica da eficácia e segurança do uso das plantas medicinais e produtos fitoterápicos (Bruning et al, 2012; Figueiredo et al, 2014).

\section{Considerações Finais}

A utilização de plantas medicinais é comum a inúmeras culturas, sendo o somatório destas a responsável pela evolução da utilização do uso de plantas medicinais como forma farmacêutica, tendo este contribuído significativamente para o surgimento das ciências médicas.

Grande parte do potencial terapêutico das plantas medicinais ainda são desconhecidos, sendo cada vez mais necessário o investimento em pesquisas de bioprospecção, visando a descoberta de novas moléculas com potencial terapêutico.

Estudos de validação quanto ao uso medicinal das plantas são de extrema importância como instrumento de validação do conhecimento tradicional, garantindo a eficácia e a segurança da utilização destes como agente terapêutico.

Considerando que grande parte da população brasileira ainda carece de condições básicas de acesso à saúde, a utilização das plantas medicinais e a sua inserção no Sistema Único de Saúde representa um grande avanço social, uma vez que contribui significativamente com a democratização do acesso à saúde, provendo às comunidades carentes uma possibilidade para a cura e o tratamento de inúmeras doenças.

\section{Agradecimentos}

Os autores agradecem a Alexandre Gomes da Silva (in memorium), Fundação de Amparo à Ciência e Tecnologia de Pernambuco (FACEPE), Coordenação de Aperfeiçoamento de Pessoal do Nível Superior (CAPES), Conselho Nacional de Desenvolvimento Científico e Tecnológico (CNPq), Ministério da Ciência, Tecnologia e Inovações (MCTI) e também para projeto licuri processo 403583/2020-9.

\section{Referências}

Albuquerque, U. P. D., \& Hanazaki, N. (2006) Ethnodirected research in the discovery of new drugs of medical and pharmaceutical interest: flaws and perspectives. Revista Brasileira de Farmacognosia, 16, 678-689.

Almeida, M. Z. (2011). Plantas medicinais: Abordagem histórica contemporânea. Editora da UFBA.

Amorim, A. M., \& Chow, F. (2016). Biodiversidade: que diversidade é essa? O exemplo das macroalgas. Instituto de Biociências, Universidade de São Paulo,

Barros, J. A. (2002) Pensando o processo saúde doença: a que responde o modelo biomédico?*. Saúde e Sociedade, $11,67-84$.

Batista, L. M., \& Valença, A. M. A (2012). Fitoterapia no Âmbito da Atenção Básica no SUS: Realidades e Perspectivas. Pesquisa Brasileira em Odontopediatria e Clínica Integrada, 12, 293-296.

Braga, Carla de Morais (2011). Histórico da utilização de plantas medicinais. Monografia (Licenciatura em Biologia) - Universidade de Brasília/Universidade Estadual de Goiás, Brasília, 2011.

Brandão, M. D. G. L., Paula-Souza, J., Grael, C.F.F., Scalon, V., Santos, A. C., Salimenha, M.F., \& Monte-Mór, R. L. (2010). Biodiversidade, uso tradicional de plantas medicinais e produção de fitoterápicos em minas gerais. In: Anais do XIV Seminário sobre a Economia Mineira. Cedeplar, Universidade Federal de Minas Gerais, 2010.

Brasil. Constituição (1988). Constituição da República Federativa do Brasil. Brasília, Senado Federal: Centro Gráfico, 1988.

Brasil. Presidência da República. Decreto $n^{\circ} 7.508$, de 28 de junho de 2011. Regulamenta a Lei no 8.080 , de 19 de setembro de 1990 , dispõe sobre a organização do Sistema Único de Saúde - SUS e dá outras providências.

Brasil. Ministério da Saúde. Secretaria de Ciência, Tecnologia e Insumos Estratégicos. Departamento de Assistência Farmacêutica e Insumos Estratégicos. Relação Nacional de Medicamentos Essenciais: RENAME 2014 Ministério da Saúde. 2015.

Brasil. Decreto n. 2.519, de 16 de Março de 1998. Promulga a Convenção sobre a Diversidade biológica, assinada no Rio de Janeiro, em 5 de Junho de 1992. 
Research, Society and Development, v. 10, n. 10, e44101018282, 2021

(CC BY 4.0) | ISSN 2525-3409 | DOI: http://dx.doi.org/10.33448/rsd-v10i10.18282

Presidência da República Federativa do Brasil. https://www.planalto.gov.br/ccivil_03/decreto/D2519.htm.

Bruning, M. C., Mosegui, G. B., \& Vianna, C. M. (2012). The use of phytotherapy and medicinal plants in primary healthcare units in the cities of cascavel and Foz do Iguaçu - Paraná: The viewpoint of health professionals. Ciencia e Saude Coletiva, 17, 2675-2685.

Bulletin Of The World Health Organization (1998). Regulatory situation of herbal medicines. A world wide review, 1998

Cabral, C., \& Pita, J. R. (2015). Ciclo de Exposições: Temas de Saúde, Farmácia e Sociedade. Catálogo. Formas e formatos dos medicamentos - A evolução das formas farmacêuticas. Universidade de Coimbra, Coimbra, 2015. https://www.uc.pt/ffuc/patrimonio_historico_farmaceutico/pu blicacoes/catalogosdeexposicoes/catalogo_2exp.pdf.

Calainho, D. B. (2005). Jesuítas e Medicina no Brasil Colonial. Tempo, (19), 61-75

Calixto, J. B. (2003). Biodiversidade como fonte de medicamentos. Ciência e Cultura, 55, 37-39

Costa, I. L. (2017). A transição da idade média para a idade moderna: uma análise crítica. Revista Tempo de Conquista, 19, 1-14

Cruz, M. M. (2014) Concepção de saúde-doença e o cuidado em saúde. In: SANTOS, E. M. (Org). Avaliação em Saúde: dos modelos teóricos à prática da avaliação de programas de controle de processos endêmicos. Fiocruz, 21-33.

Cunha, A. P. (2005). Aspectos históricos sobre plantas constituintes ativos e fitoterapia. http://www.ppmac.org/sites/default/files/aspectos_historicos.pdf.

Dias, J. P. (2005) A farmácia e a história: uma introdução à história da farmácia, da farmacologia e da terapêutica. Disciplina de História e Sociologia Da Farmácia Da Universidade de Lisboa. http://www.ufpi.br/subsiteFiles/lapnex/arquivos/files/Farmacia-e- Historia.pdf.

Farnsworth, N. R. (1980). The development of pharmacological and chemical research for application to traditional medicine in developing countries. Journal of Ethnopharmacology, 2, 173-181.

Ferreira, S. K., Cunha, I. P., Meneghim, M., \& Checchi, M. H. (2020). Política Nacional de Práticas Integrativas e Complementares no SUS. Ciência \& Saúde Coletiva, 9, 76 .

Figueiredo, C. A., Dantas Gurgel, I. G., \& Gurgel Junior, G. D. (2014). A Política Nacional de Plantas Medicinais e Fitoterápicos: construção, perspectivas e desafios. Physis: Revista da Saúde Coletiva, 24, 381-400.

Figueiredo, F. F. (2018). A agenda político-ambiental no Brasil. Revista Espaço Acadêmico, 18, 106-115.

Filgueiras, T. S., \& Peixoto, A. L. (2002). Flora e flora e vegetação do Brasil na carta de Caminha. Acta Botanica Brasílica, 16, 263-272.

Filho, V. C., \& Yunes, R. A. (1998). Estratégias para a obtenção de compostos farmacologicamente ativos a partir de plantas medicinais. Conceitos sobre modificação estrutural para otimização da atividade. Química nova, 21, 99-105.

Firmo, W. D., De Menezes, V. D., De Castro Passos, C. E., Dias, C. N., Alves, L. P., Dias, I. C., \& Olea, R. S. (2011). Contexto histórico, uso popular e concepção científica sobre plantas medicinais. Caderno de Pesquisa

Gomes, M. J. V. M., \& Reis, A. M. M. (2000). Ciências farmacêuticas: uma abordagem em farmácia hospitalar. Atheneu.

Giulietti, A. M., De Queiroz, L. P., Wanderley, M. D., \& Van Den Berg, C. A. (2005). Biodiversidade e conservação das plantas no Brasil. Megadiversidade, $1,52-61$.

Hoffmann, R., \& Anjos, M. (2018). Construção histórica do uso de plantas medicinais e sua interferência na socialização do saber popular. 4, $142-163$.

Ibiapina, W. V. et al. (2014). Inserção da fitoterapia na atenção primária aos usuários do SUS. Revista de Ciências da Saúde Nova Esperança, 12, 60-70.

Instituto Brasileiro De Geografia Estatística. (2013). Biomas do Brasil. https://www.ibge.gov.br/geociencias/informacoes-ambientais/15842biomas.html?=\&t=o-que-e.

Lévêque, C. (1999). A biodiversidade. Bauru: Ed. da Universidade Sagrado Coração, 1999.

Luz, M. T. (2005). Cultura contemporânea e medicinas alternativas: novos paradigmas em saúde no fim do século XX. Physis: Revista de Saúde Coletiva, v. 15 , p. 145-176, 2005.

Matos, F. J. A. (1994). Farmácias vivas. (2a ed.), Ed. EUFC/ Laboratório de Produtos Naturais, 260p.

Medeiros, M. (2009). Etnobotânica histórica: princípios e procedimentos. NUPPEA, 2009.

Ministério Da Saúde (2006). Política Nacional de Práticas Integrativas e Complementares (PNPIC) no Sistema Único de Saúde. http://bvsms.saude.gov.br/bvs/publicacoes/pnpic.pdf.

Miranda, G. S., Souza, S. R., Amaro, M. O., Da Rosa, M. B., \& De Carvalho, C. A. (2013) Avaliação do conhecimento etnofarmacológico da populąção de Teixeiras - MG, Brasil. Revista de Ciencias Farmaceuticas Basica e Aplicada, 34

Monteiro, S. D. C., \& Brandelli, C. L. C. (2017). Farmacobotânica: Aspectos Teóricos e Aplicação. Artmed Editora.

Neto, P. A., \& Caetano, L. C. (2005). Plantas medicinais: do popular ao científico. Universidade Federal de Alagoas, 2005.

Nogueira, R. C., Cerqueira, H. F., \& Soares, M. B. P. (2010). Patenting bioactive molecules from biodiversity: the Brazilian experience. Expert Opinion On 
Research, Society and Development, v. 10, n. 10, e44101018282, 2021

Therapeutic Patents, 20, 145-157.

Oliveira, M. J., Simoes, M. J., Sassi, C. R. (2006). Fitoterapia no sistema de saúde pública (SUS) no Estado de São Paulo, Brasil. Revista Brasileira de Plantas Medicinais, p. 39-41.

Paim, J. S. (2008). Reforma Sanitária Brasileira: contribuição para a compreensão e crítica. FIOCRUZ, 2008. http://books.scielo.org/id/4ndgv/pdf/paim9788575413593.pdf

Peixoto, A. L, Morim, M. P. (2003). Coleções botânicas: documentação da biodiversidade brasileira. Ciência e Cultura, 55, $21-24$.

Pelt, J. M. (2004). Especiarias e Ervas Aromáticas: história, botânica e culinária. Zahar.

Pereira Júnior, Raimundo Carlos (2005). Estudo fitoquímico e avaliação da potencialidade farmacológica de extratos de Crateva benthamii (Capparaceae). 2005. Dissertação (Mestrado em Biotecnologia) - Escola Superior de Ciências da Saúde, Universidade do Estado do Amazonas, Manaus, 2005.

Petrovska, B. B. (2012). Historical review of medicinal plants usage. Pharmacognosy Reviews, 6, 1.

Phillipson, J. D. (2001). Phytochemistry and medicinal plants. Phytochemistry, 53, 237-243.

Pinto, A. C., Silva, D. H., Bolzani, V. D., Lopes, N. P., Epifanio, R. D. (2002) Current status, challenges and trends on natural products in Brazil. Quimica Nova, 25. 45-61.

Pires, M. J. P. (1984) Aspectos históricos dos recursos genéticos de plantas medicinais. Rodriguésia, 35, 61-66.

Rocha, F. A., Araújo, M. F., Costa, N. D., Silva, R. P. (2015)O Uso Terapêutico Da Flora Na História Mundial. Holos. 1, 49-61.

Rodrigues, M. D., Gomide, M. (2019). Acesso à fitoterapia na atenção básica em saúde através da Análise de Redes Sociais (ARS). Redes: Revista Hispana para el análisis de redes sociales, 30, 244-253.

Rodrigues, A. C., Guedes, M. L. (2006). Utilização de plantas medicinais no Povoado Sapucaia, Cruz das Almas - Bahia. Revista Brasileira de Plantas Medicinais, 8, 1-7.

Ronan, C. A. (1987). História ilustrada da ciência. Universidade de Cambridge, v. 4.

Saad, B. (2014). Greco-Arab and Islamic Herbal Medicine: A Review. European Journal of Medicinal Plants, $249-258$.

Santos, R. L., Guimarães, G. P., Nobre, M. S., \& Portela A. D. (2011). Análise sobre a fitoterapia como prática integrativa no Sistema Único de Saúde. Revista Brasileira de Plantas Medicinais, 13, 486-491.

Saraiva, S. R., Saraiva, H. C., De Oliveira Junior, R. G.,Silva, J. C., Damasceno, C. M., Da Silva Almeida, J. R., Amorim, E. L. (2015). A implantação do programa de plantas medicinais e fitoterápicos no sistema público de saúde no brasil: uma revisão de literatura. Revista Interdisciplinar de Pesquisa e Inovação

Sheldon, J. W. (1997) Medicinal Plants: Can utilization and conservation coexist? Advances in Economy Botany, 12 , i-104.

Silva, A. F., Rabelo, M., Magalhães, M. (2014). Bioprospecção de espécies vegetais nativas do cerrado com potencial para o desenvolvimento de antimicrobianos. Circular-Técnica n. 200, Empresa de Pesquisa Agropecuária de Minas Gerais, 2014.

SILVA, Jackeline G. (2007) Avaliação do potencial farmacológico de Kalanchoe brasiliensis Cambess. 2007. Dissertação (Mestrado em Ciências Farmacêuticas) - Departamento de Ciências Farmacêuticas, Universidade Federal de Pernambuco, Recife, 2007.

Souza, S. A. (2019). Seguindo redes e ramificações de produção de conhecimentos em práticas de cura a partir do uso das Plantas Medicinas e Fitoterápicos no Município de Vitória, Anais do Seminário de Ciências Sociais, 4, 2019.

Veiga, V. F., Pinto, A. C., Maciel, M. A. (2005). Medicinal plants: Safe cure? Quimica Nova, 28, 519-528.

Viegas Jr, C., Bolzani, V. D., Barreiro, E. J. (2006). Os produtos naturais e a química medicinal moderna. Química Nova, $326-337$.

Walker, T. D. (2013). The medicines trade in the portuguese atlantic world: Acquisition and dissemination of healing knowledge from Brazil. História Social da Medicina, 26, 403-431. 